\title{
tRNA gene diversity in the three domains of life
}

\author{
Kosuke Fujishima ${ }^{1,2 *}$ and Akio Kanai ${ }^{2 *}$ \\ ${ }_{1}^{1}$ NASA Ames Research Center, Moffett Field, CA, USA \\ 2 Institute for Advanced Biosciences, Keio University, Tsuruoka, Japan
}

\section{Edited by:}

Sanjeev Kumar Srivastava, Mitchell

Cancer Institute, USA

Reviewed by:

Sandeep Kumar, SUNY Downstate

Medical Center, USA

Nikhil Tyagi, Mitchell Cancer

Institute, USA

Kaushlendra Tripathi, Mitchell

Cancer Institute, USA

\section{*Correspondence:}

Kosuke Fujishima, NASA Ames

Research Center, Building N239,

Moffett Field, CA 94035, USA

e-mail: kosuke.fujishima@nasa.gov;

Akio Kanai, Institute for Advanced

Biosciences, Keio University, 403-1

Nipponkoku, Daihoji, Tsuruoka

997-0017, Japan

e-mail:akio@sfc.keio.ac.jp
Transfer RNA (tRNA) is widely known for its key role in decoding mRNA into protein. Despite their necessity and relatively short nucleotide sequences, a large diversity of gene structures and RNA secondary structures of pre-tRNAs and mature tRNAs have recently been discovered in the three domains of life. Growing evidences of disrupted tRNA genes in the genomes of Archaea reveals unique gene structures such as, intron-containing tRNA, split tRNA, and permuted tRNA. Coding sequence for these tRNAs are either separated with introns, fragmented, or permuted at the genome level. Although evolutionary scenario behind the tRNA gene disruption is still unclear, diversity of tRNA structure seems to be co-evolved with their processing enzyme, so-called RNA splicing endonuclease. Metazoan mitochondrial tRNAs (mtRNAs) are known for their unique lack of either one or two arms from the typical tRNA cloverleaf structure, while still maintaining functionality. Recently identified nematode-specific $V$-arm containing tRNAs (nev-tRNAs) possess long variable arms that are specific to eukaryotic class II tRNA ${ }^{\text {Ser }}$ and tRNA ${ }^{\text {Leu }}$ but also decode class I tRNA codons. Moreover, many tRNA-like sequences have been found in the genomes of different organisms and viruses. Thus, this review is aimed to cover the latest knowledge on tRNA gene diversity and further recapitulate the evolutionary and biological aspects that caused such uniqueness.

Keywords: intron-containing tRNA, split tRNA, permuted tRNA, nev-tRNA, armless tRNA, RNA splicing endonuclease, co-evolution

\section{INTRODUCTION}

Transfer RNA (tRNA) is a short non-coding RNA of approximately $70-100$ bases. The principal function of tRNA is its involvement in translation machinery. Each tRNA is charged with a corresponding amino acid and delivered into the ribosome during protein biosynthesis. Currently all living organism possess tRNA molecules and thus it is known as one of the most classical RNA molecules found in nature, and it is essential for the core biological system. The recent development in the fields of genomics and transcriptomics has revealed many non-canonical tRNA genes and their structures in the three domains of life. These include tRNA gene disruption, fragmentation, rearrangement, minimization, and re-coding (Kanai, 2013). It is likely that such diversification is a consequence of the co-evolution of tRNA and its processing enzyme known as splicing endonuclease (Tocchini-Valentini et al., 2005a). RNA splicing endonuclease in Archaea recognizes and cleaves a structural motif consisting of two three-nucleotide bulge loops separated by four base pairs, known as the bulge-helix-bulge (BHB) motif (Thompson and Daniels, 1990). Structural motif and location of tRNA introns in Archaea have been affected by the change in the recognition and activity of the different unit compositions in the tRNA splicing endonucleases (Fujishima et al., 2011). Some type of disrupted tRNA genes such as split tRNA are considered as potential analogs of early tRNA, thus creating a hot debate in the field of tRNA evolution (Randau and Söll, 2008; Di Giulio, 2012). While it is still unclear how tRNA molecules originated, evolutionary biologists continue to question how these chains of ribonucleotides became involved in the context of protein synthesis, and how they influenced the evolution of these biological systems. When in the course of molecular evolution did tRNA molecule and its characteristic cloverleaf structure emerged is still an ongoing debate, however several evolutionary models representing the origin and convergence of proto-tRNA have been proposed (Weiner and Maizels, 1999; Widmann et al., 2005; Sun and Caetano-Anollés, 2007). In this review, we will recapitulate the characteristics of modern tRNA gene diversity, summarize the coevolutionary scenario of tRNA and their processing enzymes, and provide different models for the origin and evolution of early tRNA.

\section{tRNA GENE DIVERSITY IN THE THREE DOMAINS OF LIFE}

In the current era of large scale-genomics a large number of complete genome sequences are available; 2615 Bacteria, 166 Archaea, 171 Eukaryote, and 3490 Virus (March 2014; https://www.ebi.ac. uk/genomes/). tRNA genes have been long predicted computationally using a classical software tRNA-scanSE which enables one to identify $99-100 \%$ of canonical cloverleaf structure and introns at the anticodon loop with very few false positives (Lowe and Eddy, 1997). However, in 2005, a first example of trans-spliced tRNA encoded on two separate genes, so-called split tRNA was identified (Randau et al., 2005a). Two tRNA halves are bound by the complimentary leader sequence that forms the characteristic BHB motif at the tRNA exon-intron boundary. In an attempt to find structurally disrupted tRNA genes, our team developed a new tRNA prediction software SPLITS and SPLITSX (Sugahara et al., $2006,2007)$ that centers on finding multiple intron-containing 
tRNA and split tRNA through detection and removal of BHB motifs at the genome level. For similar reasons, improved version of tRNA-scanSE was recently launched (Chan et al., 2011). Based on these powerful computational approaches, a variety of non-standard tRNA genes have been found in all three domains of life and organelles (Table 1). We also summarized the tRNA gene diversity on the tree of life (Figure 1). Domain Archaea provides many of the examples of non-standard tRNAs such as multiple-intron containing tRNAs (Sugahara et al., 2008), split tRNAs (Randau et al., 2005a; Chan et al., 2011), tri-split tRNAs (Fujishima et al., 2009), and permuted tRNAs (Chan et al., 2011). Permuted tRNAs are also found in the nuclear and nucleomorph genomes of early-diverged eukaryotic algae (Soma et al., 2007; Maruyama et al., 2010). The only exception (exception to what?) is the Nematode-specific variable arm containing tRNAs (nevtRNAs) that decode alternative genetic code found in the phylum Nematoda (Hamashima et al., 2012). Many metazoan mitochondrial tRNAs are known for their armless tRNA structures lacking either one, or in extreme case, both of their arms (Ohtsuki et al., 2002; Masta and Boore, 2008). On the contrary, bacterial tRNA genes are surprisingly uniform and lack structural diversity. The only significant example found so far is the insertion of group I and II introns between nucleotides 37 and 38 of the precursor tRNA (37/38) when they are predominantly positioned adjacent to the anticodon (Paquin et al., 1997; Vogel and Hess, 2001). Similarly this location has also been known as "canonical" intron insertion site for enzymatically spliced introns in the tRNA precursors of Archaea and Eukaryotes (Abelson et al., 1998).

\section{INTRON-CONTAINING TRNA}

tRNA splicing events occur in all three domains: bacteria, archaea, and eukaryotes. In bacteria, tRNA introns are self-splicing group I introns found mostly in cyanobacteria and in few alpha- and betaproteobacteria (Reinhold-Hurek and Shub, 1992; Tanner and Cech, 1996). There are currently two group I intron families in cyanobacteria. One is found in $\mathrm{tRNA}^{\mathrm{fMet}}$ that are recently recently gain by lateral gene transfer. The other one has more ancestral origin, found at exact same position of tRNA ${ }^{\text {Leu }}$ (UAA) genes in both cyanobacteria and organelles called plastid that originated from endosymbiotic cyanobacteria (Paquin et al., 1997). The typical secondary structure of a group I intron consists of approximately 10 helical elements with roughly 100 nucleotides as central catalytic core of the intron RNA to facilitate the splicing reaction (Haugen et al., 2005). The majority of the bacterial and plastid tRNA group I introns are located at position 37/38 immediately downstream of the anticodon. Interestingly the same feature is observed for eukaryotic and archaeal tRNA introns that are relatively short in length (less than $100 \mathrm{nt}$ ) and are spliced by a series of protein enzymes called splicing endonucleases (Abelson et al., 1998). Eukaryotic and archaeal tRNA splicing share similar RNA motif recognized by orthologous splicing endonucleases, and thus origin of the tRNA introns at canonical position is assumed to be very ancestral (Kanai, 2013). Whereas, recent bioinformatics studies have revealed a number of archaeal introns located at non-canonical positions and even some tRNA genes that harbor multiple introns (Sugahara et al., 2006, 2009). There is a clear trend showing that multiple intron-containing tRNAs are prominent in the archaeal order Thermoproteales. In the most extreme cases, more than half of the tRNA genes are intervened by multiple introns (Sugahara et al., 2008). Comprehensive sequence comparison of introns among seven Thermoproteales clearly show that similar intron sequences are observed among diverse tRNA species, indicating a large-scale intron transposition occurred within this archaeal order and that could have contributed to the rapid gain of introns (Fujishima et al., 2010).

\section{SPLIT tRNA AND TRI-SPLIT tRNA}

The first example of trans-spliced tRNA was reported in 2005 by Dieter Söll's group from a highly reduced genome of an ultrasmall nanoarchaeal parasite Nanoarchaeum equitans, termed as "split tRNA" (Randau et al., 2005a). In total, 11 fragmented tRNA genes encoding $5^{\prime}$ or $3^{\prime}$-tRNA halves were determined through expression and sequencing analysis. This also included a unique case where one $3^{\prime}$-tRNA Glu half was shared by two $5^{\prime}$-tRNA ${ }^{\text {Glu }}$ halves (Randau et al., 2005b). tRNA halves are joined in trans through annealing of complimentary leader sequences and forms a relaxed BHB motif at the leader-exon boundary to be processed by the tRNA splicing endonuclease. Because of its unique features, split tRNA was first considered as a by-product of genome size reduction, however recently sequenced Nanoarchaeota Nst1 genome held no split tRNA genes, indicating that the splitting event is a specific feature of $N$. equitans and seems to reflect ongoing genome rearrangement (Podar et al., 2013). However, in 2009, our group found a new set of split tRNA genes in a free-living hyperthermophilic crenarchaeon Caldivirga maquilingensis genome without obvious trace of genome rearrangement nor reduction. We also found a new type of split tRNA consists of a maximum of three different RNA pieces thus coined as "tri-split tRNA" (Fujishima et al., 2009). Interestingly, some of these genes that carry anticodon sequence can be swapped with other genes to create synonymous tRNA, just like a jigsaw puzzle. In Figure 2, we summarized all the combinations of primary split/tri-split tRNA transcripts forming mature tRNAs in C. maquilingensis.

Split tRNA genes have also been discovered from four archaeal species belonging to the Desulfurococcales branch using an improved version of tRNA-scanSE (Chan et al., 2011). To date, total 29 trans-spliced tRNA genes corresponding to 12 anticodons have been identified in seven hyperthermophilic archaeal genomes (Table 1). The ligation sites of split tRNA gene varies, however it generally overlaps with the frequent intron insertion site of Archaea (Fujishima et al., 2010). That said, in Section Split tRNA and Intron-Containing tRNA Early or Late?, we will explain some of the possible hypotheses that explain the origin and evolution of these non-contiguous tRNAs.

\section{PERMUTED tRNA}

Permuted tRNA is another form of disrupted tRNA gene where the $3^{\prime}$ half of the tRNA lies upstream of the $5^{\prime}$ half. It was first discovered in the nuclear genome of unicellular red alga Cyanidioschyzon merolae, (Soma et al., 2007). Expression analysis revealed that the BHB motif is formed at the termini of permuted tRNA precursor, which is further spliced and ligated into a characteristic circular tRNA intermediate. This circular intermediate is then further processed at the acceptor stem, possibly 
Table 1 | List of various types of tRNA genes found in the three domains of life.

\begin{tabular}{|c|c|c|c|c|c|}
\hline \multirow[t]{2}{*}{ Name } & \multirow[t]{2}{*}{ Bacteria } & \multirow[t]{2}{*}{ Archaea } & \multicolumn{2}{|l|}{ Eukaryotes } & \multirow[t]{2}{*}{ References } \\
\hline & & & Genome & Organelles & \\
\hline $\begin{array}{l}\text { Group I intron } \\
\text { containing tRNA }\end{array}$ & $\begin{array}{l}\text { Cyanobacteria, } \\
\text { alpha-proteobacteria } \\
\text { and } \\
\text { beta-proteobacteria }\end{array}$ & - & - & $\begin{array}{l}\text { Plastids, } \\
\text { Chroloplast }\end{array}$ & $\begin{array}{l}\text { Reinhold-Hurek and } \\
\text { Shub, 1992; Tanner } \\
\text { and Cech, } 1996\end{array}$ \\
\hline $\begin{array}{l}\text { Intron-containing } \\
\text { tRNA (single) }\end{array}$ & - & All known Archaea & Most eukaryotes & - & $\begin{array}{l}\text { Abelson et al., 1998; } \\
\text { Marck and Grosjean, } \\
2003\end{array}$ \\
\hline $\begin{array}{l}\text { Intron-containing } \\
\text { tRNA (multiple) }\end{array}$ & - & $\begin{array}{l}\text { Crenarchaeota, } \\
\text { Nanoarchaeum equitans } \\
\text { (Nanoarchaeota), } \\
\text { Methanopyrus kandleri and } \\
\text { Methanothermobacter } \\
\text { thermautotrophicus } \\
\text { (Euryarchaeota), } \\
\text { Koraechaeum cryptofilim } \\
\text { (Korarchaeota), ARMAN-1 } \\
\text { and } 2\end{array}$ & $\begin{array}{l}\text { Cyanidioschyzon } \\
\text { merolae (red alga) }\end{array}$ & - & $\begin{array}{l}\text { Sugahara et al., } \\
\text { 2006, 2008, 2009; } \\
\text { Soma et al., } 2013\end{array}$ \\
\hline Split tRNA & - & $\begin{array}{l}\text { Nanoarchaeum equitans } \\
\text { (Nanoarchaeota), } \\
\text { Aerophyrum pernix, } \\
\text { Staphylothermus marinus, } \\
\text { Thermosphaera aggregans } \\
\text { and Caldivirga } \\
\text { maquilingensis } \\
\text { (Crenarchaeota) }\end{array}$ & - & - & $\begin{array}{l}\text { Randau et al., } \\
2005 a \text {; Chan et al., } \\
2011\end{array}$ \\
\hline Tri-split tRNA & - & $\begin{array}{l}\text { Caldivirga maquilingenesis } \\
\text { (Crenarchaeota) }\end{array}$ & - & - & $\begin{array}{l}\text { Fujishima et al., } \\
2009\end{array}$ \\
\hline Permuted tRNA & - & $\begin{array}{l}\text { Thermofilum pendens } \\
\text { (Crenarchaeota) }\end{array}$ & $\begin{array}{l}\text { Cyanidioschyzon } \\
\text { merolae (red alga), } \\
\text { Four prasinophyte } \\
\text { algae }\end{array}$ & $\begin{array}{l}\text { Nucleomorph in } \\
\text { Bigelowiella natans } \\
\text { (green algae) }\end{array}$ & $\begin{array}{l}\text { Soma et al., 2007; } \\
\text { Maruyama et al., } \\
\text { 2010; Chan et al., } \\
2011\end{array}$ \\
\hline $\begin{array}{l}\text { Nematode-specific } \\
\text { V-arm-containing } \\
\text { tRNA (nev-tRNA) }\end{array}$ & - & - & $\begin{array}{l}\text { Nematodes } \\
\text { (Caenorhabditis } \\
\text { elegans, C. brenneri, } \\
\text { C. briggsae, C. } \\
\text { japonica, C. remanei, } \\
\text { Pristionchus pacificus, } \\
\text { Meloidogyne } \\
\text { incognita, M. hapla) }\end{array}$ & - & $\begin{array}{l}\text { Hamashima et al., } \\
2012\end{array}$ \\
\hline Armless tRNA & - & - & - & $\begin{array}{l}\text { Most metazoan } \\
\text { mitochondria }\end{array}$ & $\begin{array}{l}\text { Okimoto and } \\
\text { Wolstenholme, } \\
\text { 1990; Ohtsuki et al., } \\
\text { 2002; Ohtsuki and } \\
\text { Watanabe, 2007; } \\
\text { Masta and Boore, } \\
2008\end{array}$ \\
\hline
\end{tabular}

by RNase $\mathrm{P}$ and tRNase $\mathrm{Z}$ for maturation (Soma et al., 2007). SPLITS has not only contributed in finding permuted tRNA in the genome of $C$. melorae but also in other diverse photosynthetic eukaryotes including Chlorophytes, a clade of unicellular green algae, and nucleomorph genome of the green alga Bigelowiella natans (Maruyama et al., 2010). A nucleomorph is a vestige of primitive algal nuclei which has undergone a process known as secondary endosymbiosis. The lack of permuted tRNA in other 


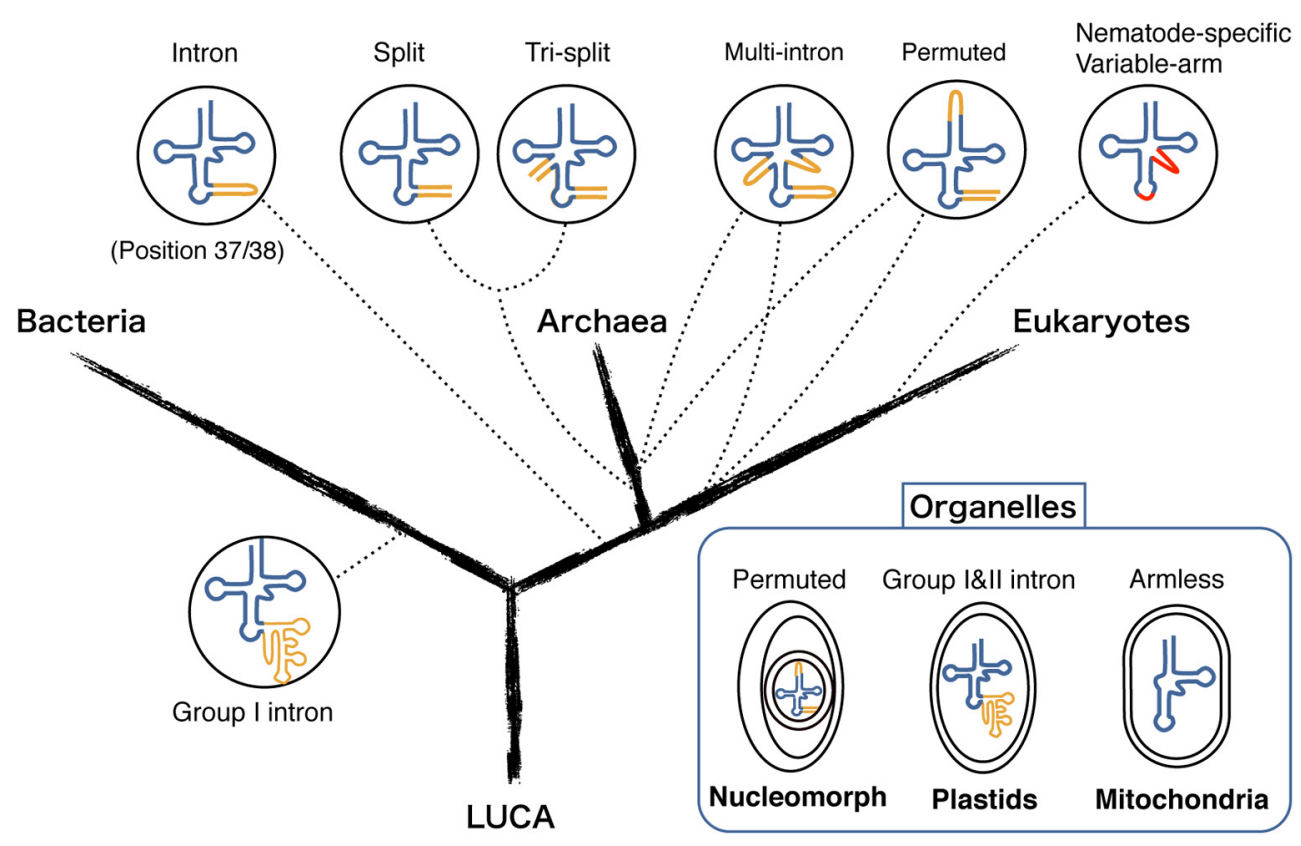

FIGURE 1 | Diversity of modern tRNA genes in the three domains of life and various organelles. Different types of tRNA genes are mapped on the phylogenetic tree of the three domains (Bacteria, Archaea, and Eukaryotes) derived from Last Universal Common
Ancestor (LUCA). Dotted lines represent the location of where each tRNA gene type is found. Intron and spacer sequences are represented in orange. Regions deviating from the standard tRNA are shown in red.

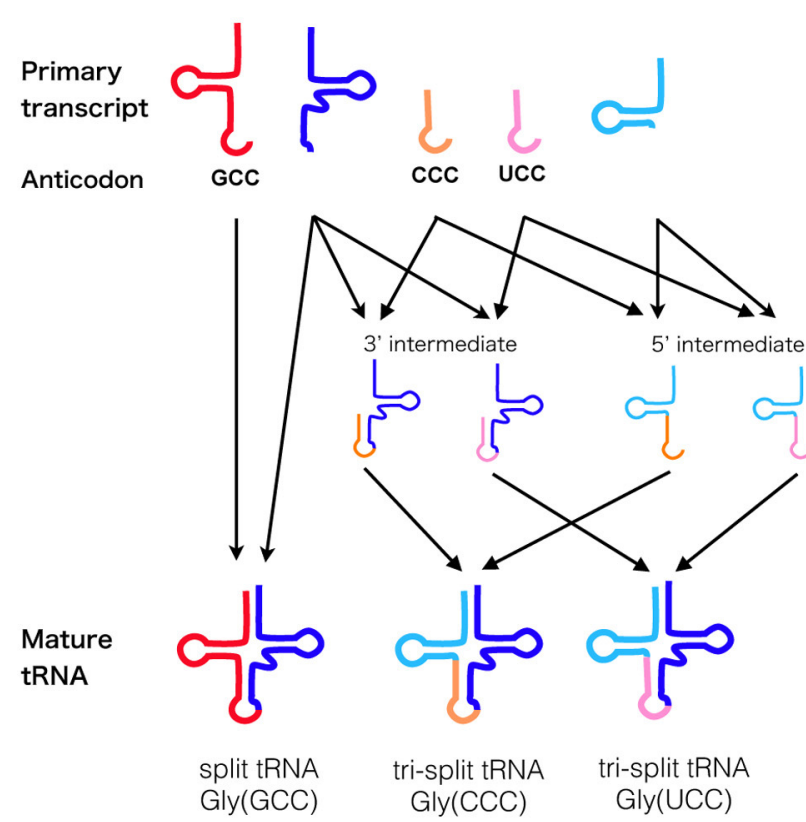

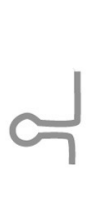<smiles>COS(=O)(=O)C(C)(C)C</smiles><smiles>C=CC(C)=O</smiles>
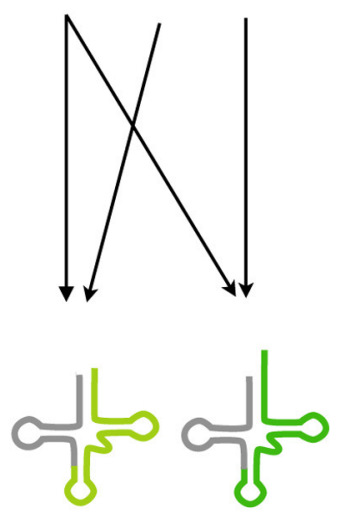

split tRNA

split tRNA Ala(UGC)
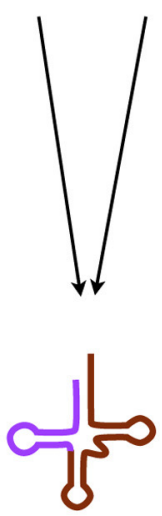

split tRNA Glu (UUC)
FIGURE 2 | Splicing pathway of tRNA fragments in

C. maquilingensis. Splicing step of the ten tRNA primary transcripts that fabricate total six split/tri-split tRNAs in archaeon
C. maquilingensis. Anticodons are denoted for the corresponding primary tRNA transcripts, and arrows represent their combination for maturation. known nucleomorph genomes and the patchy distribution of the permuted tRNA species among unicellular eukaryotes supports the gain and loss of permuted tRNA during the evolutionary stages of red and green algae (Maruyama et al., 2010). In 2011, first examples of archaeal permuted tRNA genes were found in the Crenarchaeota Thermofilum pendens genome, expanding the realm of permuted tRNAs to eukaryotes and Archaea (Chan et al., 2011). Given the accumulating phylogenomic evidence 
supporting the eocyte hypothesis, a theory where eukaryotes originate within the archaeal tree and share a sister-group with Crenarchaeota (Cox et al., 2008; Williams et al., 2013), we expect precise phylogenetic studies of permuted tRNAs and their splicing enzymes that may further strengthen this hypothesis.

\section{CO-EVOLUTION OF SPLICING ENDONUCLEASE AND TRNA}

So far, the RNA splicing endonuclease endA family is known to be the only enzyme responsible for processing the characteristic BHB motif found in precursor sequences of tRNA (Marck and Grosjean, 2003; Sugahara et al., 2007), rRNA (Tang et al., 2002), and mRNA (Yokobori et al., 2009) in Archaea, as well as removal of tRNA introns in eukaryotes. Figure 3 represents the diversity and evolutionary events that possibly occurred during the course of endA gene evolution. Currently four different types of archaeal splicing endonucleases have been identified along with their crystal structures. Both homotetrameric $\alpha_{4}$ type and homodimeric $\alpha_{2}$ type in Euryarchaeota are only capable of cleaving canonical BHB motifs (Li et al., 1998; Li and Abelson, 2000). Heterotetrameric $(\alpha \beta)_{2}$ is mostly found in Crenarchaeota (Tocchini-Valentini et al., 2005b) with a few exceptions found in Nanoarchaeota, N. equitans, and Euryarchaeota Methanopyrus kandleri (Randau et al., 2005c). Recently a fourth type of endonuclease, an unique threeunit homodimeric $\varepsilon_{2}$ type was found in ultrasmall acidophilic archaeon ARMAN-1 and 2 (Fujishima et al., 2011; Hirata et al., 2012) and it has been shown to cleave non-canonical introns (BHL and $\mathrm{hBH}$ ) inserted at various positions of tRNA (TocchiniValentini et al., 2005a; Fujishima et al., 2011). Indeed, in the

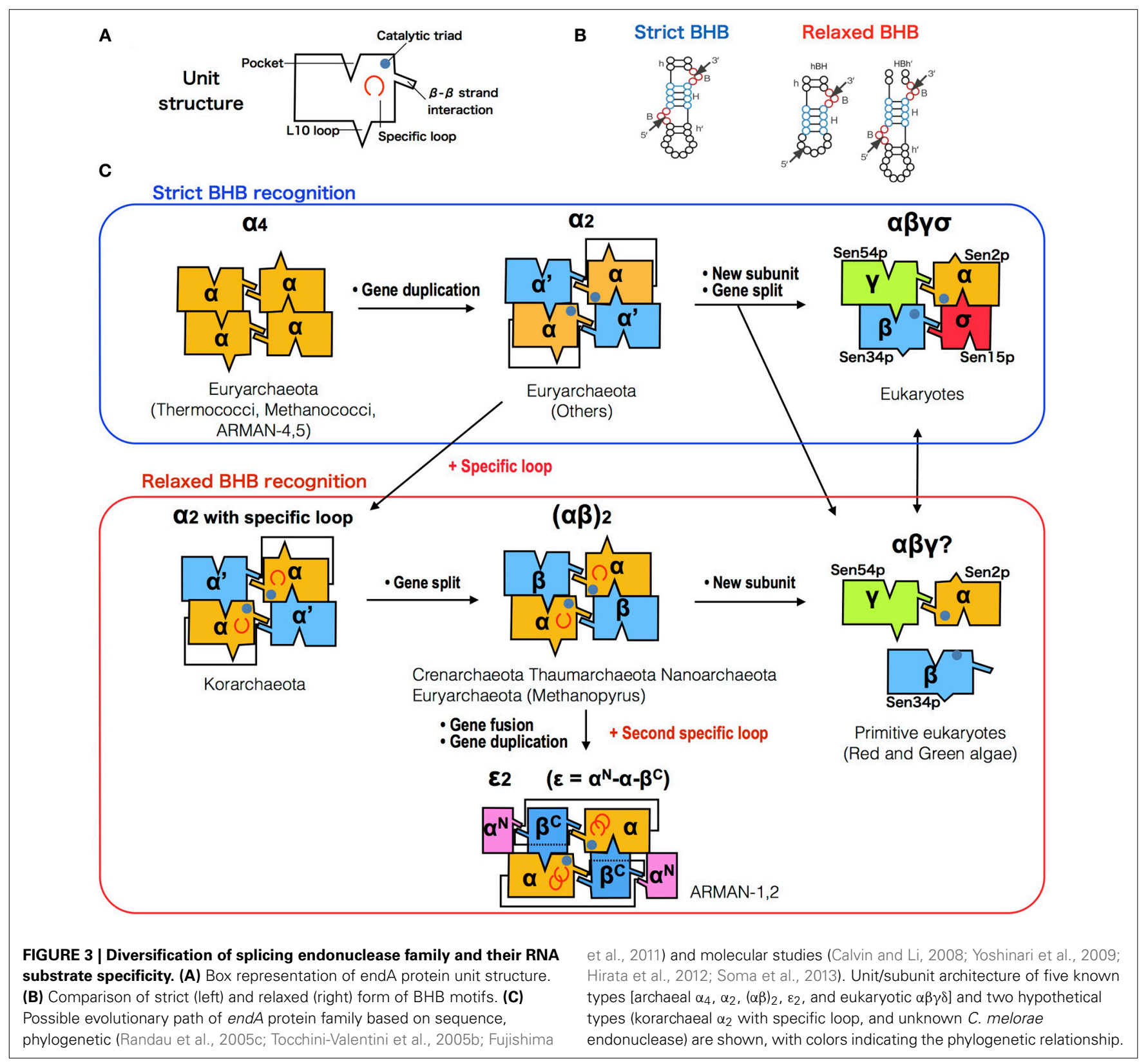


crenarchaeal order thermoproteales, large numbers of tRNA introns seem to have rapidly accumulated, generating multiple intron-containing tRNAs with a maximum of three introns. This phenomenon strongly suggest that the change of splicing endonuclease type will directly influence the tRNA gene structure (Sugahara et al., 2008). In $(\alpha \beta)_{2}$ type and $\varepsilon_{2}$ type endonucleases, an insertion of specific loops were observed and based on the structural analysis, both loops are essential for the recognition of relaxed BHB splicing motif found at the boundaries of tRNA exon and non-canonical introns (Hirata et al., 2012). These results clearly show the evolutionary path of splicing endonuclease toward acquiring broad substrate specificity, which drove the co-evolution along with tRNA genes to accept introns at various positions. Based on the sequence evidence and phylogeny of subunits, we recently proposed a novel homodimeric $\alpha_{2}$ type of archaeal endA specifically found in Korarchaeota, which shares a same "specific loop" with the $(\alpha \beta)_{2}$ type, known to be essential for the cleavage of non-canonical tRNA introns (Fujishima et al., 2011). Indeed, Korarchaeum cryptofilum is the only archaeon with $\alpha_{2}$ endonuclease that harbors tRNA genes with non-canonical introns, representing another example of the tRNA-endA coevolution. In eukaryotes, tRNA splicing endonuclease consists of four subunits Sen2p, Sen $15 p$, Sen34p, and Sen54p, in which Sen $2 p$ and Sen34p share clear sequence homology to archaeal endA (Trotta et al., 1997). The function of eukaryotic endA has been well studied in yeast, where the yeast splicing endonuclease can only strictly cleave tRNA introns located at position $37 / 38$ just after the anticodon (Reyes and Abelson, 1988). Highly conserved 50 aa core sequence shared between the two domains of life suggest a monophyletic origin of RNA splicing endonuclease as a single subunit protein forming a homotetramer known as $\alpha_{4}$ type, emerged before the divergence of Archaea and eukaryotes (Abelson et al., 1998). While protein crystal structures of the four types of archaeal endonucleases have been solved, so far only one of the four eukaryotic endA subunits sen $15 p$ has been crystalized from human homolog (Song and Markley, 2007), leaving overall orientation of the eukaryotic tRNA splicing endonuclease up to speculation. Two-hybrid experiment has previously shown that in vivo, interaction occurs between Sen $2 \mathrm{p}$ and Sen54p, and between Sen $34 \mathrm{p}$ and Sen $15 \mathrm{p}$ specifically, leading to a heterotetrameric enzyme model with Sen54p functioning as a ruler to measure the distance from the tRNA mature domain (Trotta et al., 1997). However, the genome sequence of primitive red algae $C$. melorae and green algae harbors highly-disrupted tRNA genes including permuted tRNA and multiple intron-containing tRNA. Only three homologs, cmSen $2 \mathrm{p}, \mathrm{cmSen} 34 \mathrm{p}$, and cmSen $54 \mathrm{p}$ have been identified from the $C$. melorae genome (Tocchini-Valentini and Tocchini-Valentini, 2012). More recently, a yeast-two hybrid experiment revealed a reciprocal interaction between $\mathrm{cmSen} 2$ and cmSen54, however cmSen34p did not interact with either of the two subunits suggesting a distinct complex formation and splicing machinery from canonical eukaryotic heterotetramer (Soma et al., 2013).

\section{NEV-tRNA (NEMATODE-SPECIFIC V-ARM-CONTAINING tRNAs)}

Mature tRNA can be grouped into two distinct classes (I and II) based on the presence of the long variable arm (V-arm) located between the anticodon arm and the T-arm (Brennan and
Sundaralingam, 1976). This V-arm has known to be specific for tRNA $^{\text {Ser }}$, tRNA ${ }^{\text {Leu }}$, and bacterial tRNA ${ }^{\text {Tyr }}$, and it plays an important role in the recognition of cognate aminoacyl tRNA synthetase (aaRS) (Tocchini-Valentini et al., 2000). However, comprehensive analysis of 46 diverse eukaryotic genomes revealed over 100 novel class II tRNAs in six nematodes genomes with a non-canonical anticodon, coined as nematode-specific V-arm-containing tRNA (nev-tRNA) (Hamashima et al., 2012). Comparative sequence analysis of nev-tRNA has shown that aaRS recognition elements in the $\mathrm{V}$-arm are similar to that of class II tRNA. Indeed, aminoacylation assay and in vivo translation experiment have shown that nev-tRNA ${ }^{\text {Gly }}$ can decode Gly(GGG) codon as leucine. Expression levels of two nev-tRNA ${ }^{\text {Gly }}$ (CCC) and nev-tRNA ${ }^{\text {Ile }}$ (UAU) turned out to be very low, compared to that of canonical tRNAs in C. elegans. Moreover, codons that nev-tRNAs correspond to are mostly rare codons and comprise less than $20 \%$ of the synonymous codons, suggesting it will not drastically influence the proteome (Hamashima et al., 2012). Recently we have identified additional nev-tRNAs in two plant-parasitic nematodes belonging to genus Meloidogyne. Similar anticodon alternation has been reported in other organisms including primates, Drosophila, yeast, and Enterobacteria, in which half of the cases were involved in switching the codon identity (Rogers and Griffiths-Jones, 2014). In extreme cases, anticodon shift results in assigning an alternative genetic code in certain species, such as the stop codons UGA or UAA being reassigned to various sense codons in Mycoplasma, certain ciliated protozoans, and peritrich species (Hamashima and Kanai, 2013).

\section{MITOCHONDRIAL ARMLESS tRNA}

Mitochondria are an essential organelle in eukaryotes, generating most of the cell's energy supply as ATP. It is generally accepted that mitochondria originated from within the bacterial phylum $\alpha$-Proteobacteria and underwent an endosymbiotic event (Gray, 2012). With few exceptions, animal mitochondrial genomes carry over 20 tRNA genes that are distinct from their host cell tRNA (Boore, 1999). However, some nucleus-encoded tRNAs have to be imported from the cytosol to complete the mitochondrial translation (Salinas et al., 2012). Mitochondria have also developed their own variant genetic codes from the universal genetic code, repeatedly and independently in various eukaryotic taxa (Knight et al., 2001). Currently a compilation of over 30,525 mitochondrial tRNA (mt tRNA) sequences from 1418 fully sequenced metazoan mitochondrial RefSeq genomes are registered in the mitotRNAdb (http://mttrna.bioinf.uni-leipzig.de) (Jühling et al., 2009). Most mt tRNAs possess a canonical cloverleaf structure, however extreme examples of truncated mt tRNAs have been identified in some metazoan mitochondria (Ohtsuki et al., 2002; Masta and Boore, 2008). D-armless and T-armless $\mathrm{mt}$ tRNAs were first identified in two nematode worms C. elegans and Ascaris summ (Okimoto and Wolstenholme, 1990). Later, a significant number of T-armless mt tRNAs were found in six different eumetazoa phylum including Nematodes and Arthropods (Ohtsuki and Watanabe, 2007). On the contrary, mammalian mitochondria possess only the cloverleaf and D-armless tRNA, and so far truncated mt tRNA have not been observed in either plants or fungi, suggesting that D-armless tRNA first emerged after the branching of metazoa and more recently T-armless $\mathrm{mt}$ 
tRNA arose in independent branches of eumetazoa (Ohtsuki and Watanabe, 2007). It has been speculated that the main cause of such diversification is due to the subfunctionalization of elongation factor EF-Tu. In C. elegans, mitochondrial DNA encodes two EF-Tu homologs, EF-Tu1 and EF-Tu2, which exclusively recognize aminoacylated $\mathrm{T}$-armless and $\mathrm{D}$-armless $\mathrm{mt}$ tRNAs, respectively (Arita et al., 2006). Given the fact that in mammalian mitochondria, cloverleaf and D-armless tRNAs are recognized by a single $\mathrm{mt} \mathrm{EF-Tu}$ that resembles bacterial type (Andersen et al., 2000), gene duplication of EF-Tu leading to a subfunctionalizion of EF-Tu1 to recognize T-armless mt RNA should have contributed in the co-evolution of mt tRNA species, allowing their extreme truncation (Arita et al., 2006).

\section{ORIGIN AND EVOLUTION OF TRNA-MOLECULAR EVIDENCE AND EVOLUTIONARY SCENARIO}

tRNA is unique for its capability to bridge information from nucleotide polymer (RNA) to amino acid polymer (protein). Decoding of mRNA information by tRNA is governed by triplet codon-anticodon base pairing, and each codon corresponds to one of the 20 standard proteinogenic amino acids that all living organism share in common. These amino acids are correctly charged onto the $3^{\prime}$-adenosine terminal of the tRNA molecule by aaRSs. Currently two evolutionary unrelated classes of tRNA synthetases (Class I and Class II) are known (Wolf et al., 1999), and surprisingly for many of these aaRSs, anticodon arm is not necessary for aminoacylation, capable of charging short RNA minihelices and duplexes that including the minimal $12 \mathrm{bp}$ acceptor arm-T $\Psi \mathrm{C}$ stem loop, known to be the top half of tRNA (Francklyn and Schimmel, 1989, 1990). This finding led to the theory of "operational code," which in the early stage of tRNA evolution, identity was solely embedded in the simple minihelix RNA which later became the acceptor stem of tRNA (Schimmel et al., 1993). Hence, in this chapter, we will feature major findings and theories that lead to a plausible scenario for the origin and evolution of tRNA molecule.

\section{MINIMAL AMINOACYL RIBOZYME}

The essentiality of tRNA lies in its core function of $2^{\prime}-3^{\prime}$ aminoacylation. Modern aaRS catalyze this reaction as a two-step reaction (1) Amino acid activated by ATP, forming an intermediate aminoacyl adenylate (aa-AMP), (2) Aminoacyl group is transferred from the adenylate to the tRNA $3^{\prime}$-terminal adenosine nucleotide. However, modern aaRSs are the product of aminoacylation-based translation and thus researchers have been seeking for a more primitive chemical path that eventually led to this sophisticated machinery. For example, it has been shown that high energy aminoacyl adenylate can be formed from ATP and amino acids under prebiotic conditions (Paecht-Horowitz and Katchalsky, 1973). Furthermore, an in vitro evolution experiment has achieved in selecting a ribozyme capable of selfaminoacylating its own $5^{\prime}$-hydroxyl group and transferring the aminoacyl group to the $3^{\prime}$-end of other RNA, supporting the idea that aminoacyl-tRNA synthetase ribozymes playing a important role in the RNA world (Lee et al., 2000). The most extreme example of aminoacylating ribozyme to date, is a 5-nt ribozyme discovered though radical minimization of $\mathrm{C} 3$ ribozyme that self-aminoacylates (Turk et al., 2010), this ultrasmall ribozyme initially trans-phenylalanylates a complementary 4-nt RNA selectively (Figure 4A). The transfer reaction occurs regiospecifically from the phenylalanine atom to ribose $2^{\prime}$-hydroxyl, forming an ester bond between amino acid and RNA that is identical to the modern aminoacylated tRNA. This discovery implies that short ribonucleotides can aminoacylate its counterpart to produce peptidyl-RNA, which can be interpreted as the minimal form of tRNA that could have originated in the very early stage of life.

\section{MINIHELIX MODEL}

One of the plausible models for the early stage of tRNA evolution is known as a minihelix or a minigene that forms a hairpin structure (Figure 4B). This concept comes from the fact that L-shaped tertiary structure can be distinguished into two halves, where only the top part of tRNA (acceptor stem $+\mathrm{T} \Psi \mathrm{C}$ arm) is recognized by many of the modern tRNA synthetases (Schimmel and Ribas de Pouplana, 1995). The identity of tRNA is still embedded within the acceptor stem, generally depending on position discriminator base N73 and few base pairs downstream, termed the operational RNA code (Schimmel et al., 1993). Along with the discovery of minimal self-aminoacylating ribozymes presented in the previous section, presumably aminoacylated minihelix originated in an environment where short RNA oligos and amino acids co-existed, prior to the emergence of the ribosome. Tamura and Schimmel demonstrated that D-ribose chirality of RNA minihelix exhibited a clear preference for charging L-amino acids as opposed to D-amino acids (Tamura and Schimmel, 2004), indicating that aminoacylation of RNA could have been the key toward modern protein homochirality. Consequently, ribozyme-based aminoacylation was eventually substituted by more efficient and less promiscuous homochiral protein-based aminoacylation.

\section{GENOMIC-TAG HYPOTHESIS AND TRNA-LIKE STRUCTURE}

The top half of modern tRNAs is recognized by RNase P, CCAadding enzyme, and tRNA synthetases which are all related to the maturation of tRNA molecules function in protein synthesis. Various positive strand RNA viruses in plants possess tRNA-like structures (TLSs) at the $3^{\prime}$-end of their genome sequences that are functional mimics of tRNA (Dreher, 2009) (Figure 4C). They fall into three types, mimicking the tRNA function of specific aminoacylation by valine, histidine, or tyrosine at the $3^{\prime}$ end of pseudoknotted aminoacyl acceptor stem (Pleij et al., 1985). Surprisingly their roles are diverse; tRNA mimicry providing translational enhancement, presentation of minus strand promoter elements for RNA replicase recognition, recruitment of host CCA nucleotidyltransferase as a 3 -telomere, and in vitro packaging of the viral genome (Dreher, 2010). Similar trend in using TLS for replicator element can also be seen for most retroviral RNA genomes and long terminal repeat (LTR)retrotransposons, in which annealing of the top half tRNA to the primer binding site initiates the reverse transcription (Le Grice, 2003). The growing evidence of tRNA elements involved in RNA and DNA replication has therefore led to the idea of the "Genomic Tag Hypothesis" noting that tRNA-like structural motifs initially 


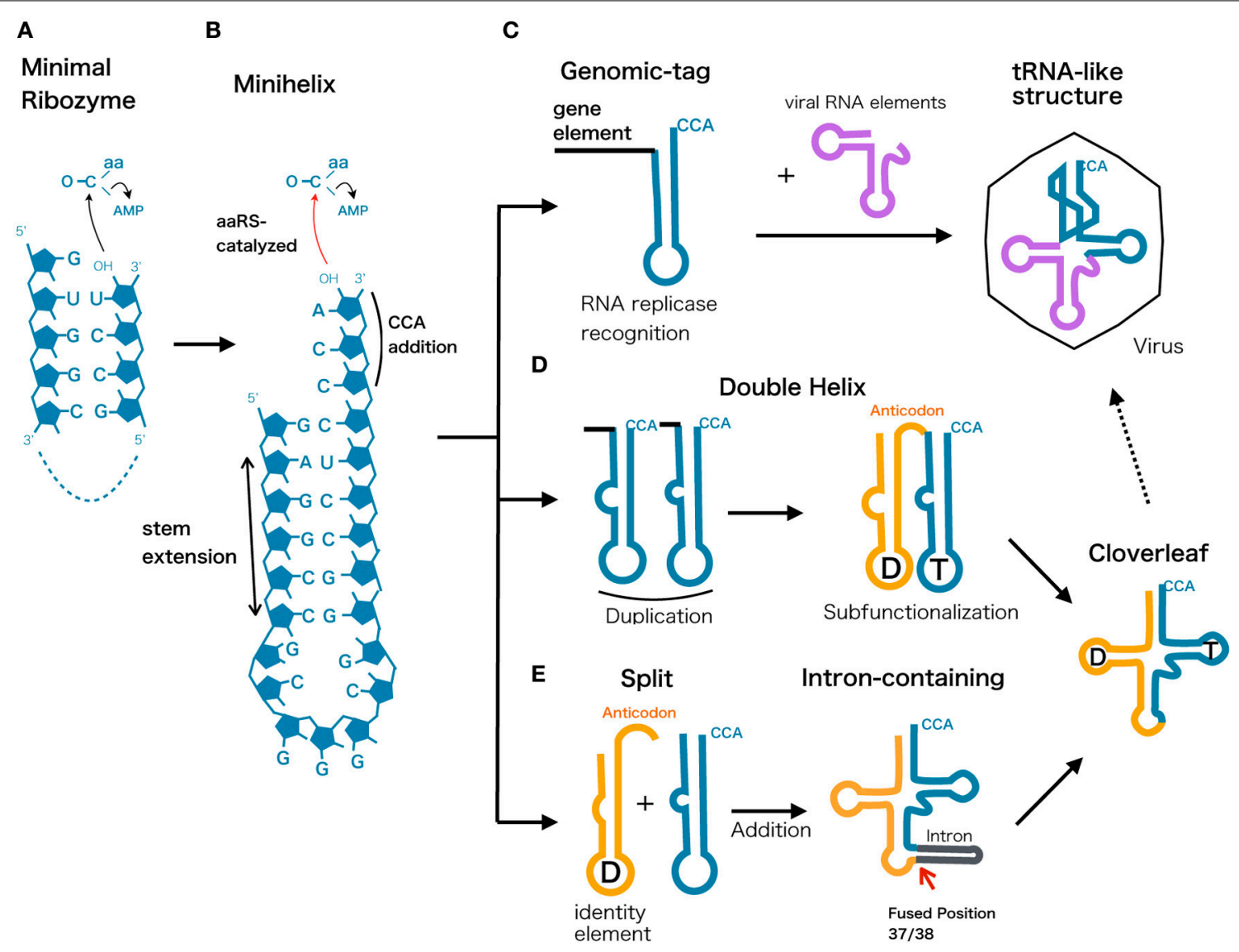

FIGURE 4 | Possible evolutionary scenarios from ancestral ribozyme to modern tRNA. The evolutionary scenarios of tRNA molecule represented along with proposed primordial models. (A) Minimal ribozyme that catalyzes and generates short peptidyl-RNA, (B) minihelix harboring the $3^{\prime}$-CCA terminal sequence, (C) Genomic-tag hypothesis showing the recognition of minihelix by RNA replicase. This functionality could have been retained and integrated into the tRNA-like structure found in modern viral genomes. (D) Double helix model assuming a duplication event that led to a subfunctionalization of two hairpin loops that are later diversified into D-arm and anticodon plus T-arm. (E) Split tRNA early model, showing a fusion of different minihelices that eventually led to modern cloverleaf. This is based on the fact that tRNA introns are universally and predominantly found at position 37/38 and that intron could be a reminiscent scar of gene fusion. evolved as a $3^{\prime}$ terminal motif that tagged RNA genomes for replication in the RNA world before the advent of protein synthesis (Weiner and Maizels, 1999).

\section{DOUBLE HELIX MODEL}

From a thermodynamic perspective, modern cloverleaf structure of tRNA is not a minimum free energy state but rather a local minimum structure that is reinforced by multiple post transcriptional modification (Wuchty et al., 1999). Similarities between nucleotides at comparable positions within the $5^{\prime}$ and $3^{\prime}$ halves of the tRNA molecule have often been taken as evidence that the modern cloverleaf structure arose through direct duplication of a hairpin (Tanaka and Kikuchi, 2001; Widmann et al., 2005) (Figure 4D). Indeed, one of the suboptimal structures of tRNA is a double helix structure that closely resembles a duplicate form of a minihelix and this structural feature was confirmed by in vitro cleavage of tRNA using RNase P (Hori et al., 2000). One possible scenario is that after the duplication event, the latter top half with $3^{\prime}$-CCA terminal continued its role as a recognition RNA element, while bottom half underwent subfunctionalization to provide anticodon and sequence diversity to coevolve with variants of tRNA synthetases.

\section{SPLIT tRNA AND INTRON-CONTAINING tRNA EARLY OR LATE?}

The symmetric form of tRNA is also considered as a consequence of two separate hairpin RNAs fused to form a modern tRNA structure (Figure 4E). The evidence showing that intron sequence is universally found at position $37 / 38$ just after the anticodon indicates a scenario that introns could be a trace of the assembled two hairpin RNA genes (Di Giulio, 2006). The difference from the duplicated double helix model is that the later added bottom half of the tRNA (D-arm + anticodon) could be evolutionary distinct from the top half. We have previously suggested the possibility of separate origin of $5^{\prime}$ and $3^{\prime}$ tRNA halves based on the sequence similarity and diversity in archeal tRNAs (Fujishima et al., 2008). Furthermore, based on phylogenetic studies of both structural and statistical characteristics of over 500 tRNA molecules suggests that the bottom half of the tRNA was added later in time (between 3 to 4 billion years ago) to the ancient top half, which grew by slow substructural accretion (Sun and Caetano-Anollés, 
2007). Currently, split tRNA genes that separately encode the $5^{\prime}$ and $3^{\prime}$ halves of tRNAs are found in several archaeal genomes (see Section Split tRNA and Tri-Split tRNA for detail). Their split positions and flanking sequences resemble the intron in related species, indicating a strong evolutionary link between split tRNA and intron-containing tRNA (Fujishima et al., 2008, 2009). It is likely that the split tRNA that we currently see in several archaeal genomes is a recently acquired trait. For example, recently the sequenced genome of a first terrestrial hyperthermophilic member of nanoarchaeota Nst1 possess not even a single split tRNA gene (Podar et al., 2013). Interestingly, while $N$. equitans possess $(\alpha \beta)_{2}$ type endonucleases capable of processing non-canonical introns and split tRNA, Nst1 genome encodes $\alpha_{4}$ type that can only cleave canonical introns. Accordingly, acquisition of $(\alpha \beta)_{2}$ type may have allowed tRNA gene fragmentation to occur and linger in N. equitans genome (Podar et al., 2013). Indeed, we have recently found evidence of ongoing tRNA gene disruption from a community genomic library prepared from a Caldiarchaeum subterraneum-dominated microbial mat (Sugahara et al., 2012). A heterogenic clone library revealed a putative DNA recombinase coding a $1.8 \mathrm{~kb}$ DNA insertion separating the tRNA fragment and the tRNA gene, a feature that is frequently found at the integration site of mobile elements, such as conjugative plasmids and viruses. Randau and Söll have earlier suggested that split tRNA genes present a strategy for impeding the viral integration or insertion of other mobile elements into canonical tRNA genes (Randau and Söll, 2008). A similar conclusion was reached by Chen et al., based on the fact that $5^{\prime}$ and $3^{\prime}$ halves of pre-tRNA ${ }^{\text {Asp }}$ (GUC) are located adjacent to each other in two related archaea $A$. pernix and $T$. aggregans, indicating two different local genome rearrangement events that occur at the same position in the same tRNA (Chan et al., 2011). On the other hand, Di Giulio interpreted this evidence from a different perspective that the current split genes we see in Archaea may represent the transition stage through which the evolution of tRNA molecule have passed (Di Giulio, 2009). He also explains that the discrepancy of having interruption at non-canonical position of some split tRNA is expected, and compatible with the split early model where assembly of the two hairpin-like structures may also have built structures to tinker with cloverleaf structure (Di Giulio, 2012).

\section{CONCLUSIONS}

In this review, we have recapitulated the diversity of gene orientation and RNA structures of modern tRNAs in three domains of life, organelles and viruses. We also focused on the co-evolution of tRNA and their splicing endonucleases, and discussed how subfunctionalization of the enzyme could shape tRNA gene arrangement by allowing the tRNA gene to accept introns at various positions as well as allowing gene fragmentation and permutation. The origin of the tRNA intron is still under debate since intron located at canonical position $37 / 38$ is conserved between Archaea and Eukaryotes and thus represents an ancestral trait, while non-canonical introns are likely added recently due to the change in the functionality of RNA splicing endonuclease. Canonical positioning is a plausible explanation of the hypothesis that modern tRNA arose from simple minihelix RNA through gene duplication/fusion. There is also growing evidence of virus or transposable elements involved in the recent addition of the tRNA intron. It will be important to collect snapshots of the tRNA intron insertion event through sequence analysis to reveal the precise molecular mechanism. Lastly, the charm of tRNA research lies in its potential of shaping the genetic code. There are examples of re-coding in mitochondria and higher eukaryotes possibly driven by tRNA gene multiplication, which tends to target the rare codons to avoid impact on proteome. Further accumulation of genomic and transcriptomic sequence data will likely provide further examples of modern tRNA diversity as well as insight into the origin and evolution of this essential molecule that is deeply involved in the rise of modern genetic system.

\section{ACKNOWLEDGMENTS}

We thank Dr. Akira Hirata (Ehime University, Japan) for providing cartoon structures for splicing endonuclease. We thank Ryan Kent (NASA Ames Research Center, USA) for proofreading this manuscript. Finally we thank all the members of the RNA group at the Institute for Advanced Biosciences, Keio University, Japan, for their insightful discussions.

\section{REFERENCES}

Abelson, J., Trotta, C. R., and Li, H. (1998). tRNA splicing. J. Biol. Chem. 273, 12685-12688. doi: 10.1074/jbc.273.21.12685

Andersen, G. R., Thirup, S., Spremulli, L. L., and Nyborg, J. (2000). High resolution crystal structure of bovine mitochondrial EF-tu in complex with GDP. J. Mol. Biol. 297, 421-436. doi: 10.1006/jmbi.2000.3564

Arita, M., Suematsu, T., Osanai, A., Inaba, T., Kamiya, H., Kita, K., et al. (2006). An evolutionary 'intermediate state' of mitochondrial translation systems found in Trichinella species of parasitic nematodes: co-evolution of tRNA and EF-Tu. Nucleic Acids Res. 34, 5291-5299. doi: 10.1093/nar/gkl526

Boore, J. L. (1999). Animal mitochondrial genomes. Nucleic Acids Res. 27, 1767-1780. doi: 10.1093/nar/27.8.1767

Brennan, T., and Sundaralingam, M. (1976). Structure, of transfer RNA molecules containing the long variable loop. Nucleic Acids Res. 3, 3235-3252. doi: 10.1093/nar/3.11.3235

Calvin, K., and Li, H. (2008). RNA-splicing endonuclease structure and function. Cell. Mol. Life Sci. 65, 1176-1185. doi: 10.1007/s00018-008-7393-y

Chan, P. P., Cozen, A. E., and Lowe, T. M. (2011). Discovery of permuted and recently split transfer RNAs in Archaea. Genome Biol. 12:R38. doi: 10.1186/gb2011-12-4-r38

Cox, C. J., Foster, P. G., Hirt, R. P., Harris, S. R., and Embley, T. M. (2008). The archaebacterial origin of eukaryotes. Proc. Natl. Acad. Sci. U.S.A. 105, 20356-20361. doi: 10.1073/pnas.0810647105

Di Giulio, M. (2006). The non-monophyletic origin of the tRNA molecule and the origin of genes only after the evolutionary stage of the last universal common ancestor (LUCA). J. Theor. Biol. 240, 343-352. doi: 10.1016/j.jtbi.2005.09.023

Di Giulio, M. (2009). Formal proof that the split genes of tRNAs of Nanoarchaeum equitans are an ancestral character. J. Mol. Evol. 69, 505-511. doi: 10.1007/s00239-009-9280-z

Di Giulio, M. (2012). The 'recently' split transfer RNA genes may be close to merging the two halves of the tRNA rather than having just separated them. J. Theor. Biol. 310, 1-2. doi: 10.1016/j.jtbi.2012.06.022

Dreher, T. W. (2009). Role of tRNA-like structures in controlling plant virus replication. Virus Res. 139, 217-229. doi: 10.1016/j.virusres.2008.06.010

Dreher, T. W. (2010). Viral tRNAs and tRNA-like structures. Wiley Interdiscip. Rev. RNA 1, 402-414. doi: 10.1002/wrna.42

Francklyn, C., and Schimmel, P. (1989). Aminoacylation of RNA minihelices with alanine. Nature 337, 478-481. doi: 10.1038/337478a0

Francklyn, C., and Schimmel, P. (1990). Enzymatic aminoacylation of an eightbase-pair microhelix with histidine. Proc. Natl. Acad. Sci. U.S.A. 87, 8655-8659. doi: 10.1073/pnas.87.21.8655

Fujishima, K., Sugahara, J., Kikuta, K., Hirano, R., Sato, A., Tomita, M., et al. (2009). Tri-split tRNA is a transfer RNA made from 3 transcripts that provides insight 
into the evolution of fragmented tRNAs in archaea. Proc. Natl. Acad. Sci. U.S.A. 106, 2683-2687. doi: 10.1073/pnas.0808246106

Fujishima, K., Sugahara, J., Miller, C. S., Baker, B. J., Di Giulio, M., Takesue, K., et al. (2011). A novel three-unit tRNA splicing endonuclease found in ultrasmall Archaea possesses broad substrate specificity. Nucleic Acids Res. 39, 9695-9704. doi: $10.1093 /$ nar/gkr692

Fujishima, K., Sugahara, J., Tomita, M., and Kanai, A. (2008). Sequence evidence in the archaeal genomes that tRNAs emerged through the combination of ancestral genes as $5^{\prime}$ and $3^{\prime}$ tRNA halves. PLoS ONE 3:e1622. doi: 10.1371/journal.pone. 0001622

Fujishima, K., Sugahara, J., Tomita, M., and Kanai, A. (2010). Large-scale tRNA intron transposition in the archaeal order Thermoproteales represents a novel mechanism of intron gain. Mol. Biol. Evol. 27, 2233-2243. doi: 10.1093/molbev/msq111

Gray, M. W. (2012). Mitochondrial evolution. Cold Spring Harb. Perspect. Biol. 4:a011403. doi: 10.1101/cshperspect.a011403

Hamashima, K., Fujishima, K., Masuda, T., Sugahara, J., Tomita, M., and Kanai, A. (2012). Nematode-specific tRNAs that decode an alternative genetic code for leucine. Nucleic Acids Res. 40, 3653-3662. doi: 10.1093/nar/gkr1226

Hamashima, K., and Kanai, A. (2013). Alternative genetic code for amino acids and transfer RNA revisited. Biomol. Concepts. 4, 309-318. doi: 10.1515/bmc-20130002

Haugen, P., Simon, D. M., and Bhattacharya, D. (2005). The natural history of group I introns. Trends Genet. 21, 111-119. doi: 10.1016/j.tig.2004.12.007

Hirata, A., Fujishima, K., Yamagami, R., Kawamura, T., Banfield, J. F., Kanai, A., et al. (2012). X-ray structure of the fourth type of archaeal tRNA splicing endonuclease: insights into the evolution of a novel three-unit composition and a unique loop involved in broad substrate specificity. Nucleic Acids Res. 40, 10554-10566. doi: 10.1093/nar/gks826

Hori, Y., Baba, H., Ueda, R., Tanaka, T., and Kikuchi, Y. (2000). In vitro hyperprocessing of Drosophila tRNAs by the catalytic RNA of RNase P the cloverleaf structure of tRNA is not always stable? Eur. J. Biochem. 267, 4781-4788. doi: 10.1046/j.1432-1327.2000.01534.x

Jühling, F., Mörl, M., Hartmann, R. K., Sprinzl, M., Stadler, P. F., and Pütz, J. (2009). tRNAdb 2009: compilation of tRNA sequences and tRNA genes. Nucleic Acids Res. 37, D159-D162. doi: 10.1093/nar/gkn772

Kanai, A. (2013). "Molecular evolution of disrupted transfer rna genes and their introns in archaea," in Evolutionary Biology: Exobiology and Evolutionary Mechanisms, ed P. Pontarotti (Berlin, Heidelberg: Springer), 181-193.

Knight, R. D., Freeland, S. J., and Landweber, L. F. (2001). Rewiring the keyboard: evolvability of the genetic code. Nat. Rev. Genet. 2, 49-58. doi: $10.1038 / 35047500$

Lee, N., Bessho, Y., Wei, K., Szostak, J. W., and Suga, H. (2000). Ribozyme-catalyzed tRNA aminoacylation. Nat. Struct. Biol. 7, 28-33. doi: 10.1038/71225

Le Grice, S. F. J. (2003). 'In the Beginning': initiation of minus strand DNA synthesis in retroviruses and LTR-containing retrotransposons. Biochemistry 42, 14349-14355. doi: 10.1021/bi030201q

Li, H., and Abelson, J. (2000). Crystal structure of a dimeric archaeal splicing endonuclease. J. Mol. Biol. 302, 639-648. doi: 10.1006/jmbi.2000.3941

Li, H., Trotta, C. R., and Abelson, J. (1998). Crystal structure and evolution of a transfer RNA splicing enzyme. Science 280, 279-284. doi: 10.1126/science.280.5361.279

Lowe, T. M., and Eddy, S. R. (1997). tRNAscan-SE: a program for improved detection of transfer RNA genes in genomic sequence. Nucleic Acids Res. 25, 955-964. doi: $10.1093 / \mathrm{nar} / 25.5 .0955$

Marck, C., and Grosjean, H. (2003). Identification of BHB splicing motifs in intron-containing tRNAs from 18 archaea: evolutionary implications. RNA 9, 1516-1531. doi: 10.1261/rna.5132503

Maruyama, S., Sugahara, J., Kanai, A., and Nozaki, H. (2010). Permuted tRNA genes in the nuclear and nucleomorph genomes of photosynthetic eukaryotes. Mol. Biol. Evol. 27, 1070-1076. doi: 10.1093/molbev/msp313

Masta, S. E., and Boore, J. L. (2008). Parallel evolution of truncated transfer RNA genes in arachnid mitochondrial genomes. Mol. Biol. Evol. 25, 949-959. doi: 10.1093/molbev/msn051

Ohtsuki, T., Kawai, G., and Watanabe, K. (2002). The minimal tRNA: unique structure of Ascaris suum mitochondrial tRNA(Ser)(UCU) having a short T arm and lacking the entire D arm. FEBS Lett. 514, 37-43. doi: 10.1016/S00145793(02)02328-1
Ohtsuki, T., and Watanabe, Y.-I. (2007). T-armless tRNAs and elongated elongation factor Tu. IUBMB Life 59, 68-75. doi: 10.1080/15216540701218722

Okimoto, R., and Wolstenholme, D. R. (1990). A set of tRNAs that lack either the T psi $\mathrm{C}$ arm or the dihydrouridine arm: towards a minimal tRNA adaptor. $E M B O$ J. 9, 3405-3411.

Paecht-Horowitz, M., and Katchalsky, A. (1973). Synthesis of amino acyladenylates under prebiotic conditions. J. Mol. Evol. 2, 91-98. doi: 10.1007/BF016 53989

Paquin, B., Kathe, S. D., Nierzwicki-Bauer, S. A., and Shub, D. A. (1997). Origin and evolution of group I introns in cyanobacterial tRNA genes. J. Bacteriol. 179, 6798-6806.

Pleij, C. W., Rietveld, K., and Bosch, L. (1985). A new principle of RNA folding based on pseudoknotting. Nucleic Acids Res. 13, 1717-1731. doi: 10.1093/nar/13.5.1717

Podar, M., Makarova, K. S., Graham, D. E., Wolf, Y. I., Koonin, E. V., and Reysenbach, A.-L. (2013). Insights into archaeal evolution and symbiosis from the genomes of a nanoarchaeon and its inferred crenarchaeal host from Obsidian Pool, Yellowstone National Park. Biol. Direct 8:9. doi: 10.1186/17456150-8-9

Randau, L., Calvin, K., Hall, M., Yuan, J., Podar, M., Li, H., et al. (2005c). The heteromeric Nanoarchaeum equitans splicing endonuclease cleaves noncanonical bulge-helix-bulge motifs of joined tRNA halves. Proc. Natl. Acad. Sci. U.S.A. 102, 17934-17939. doi: 10.1073/pnas.0509197102

Randau, L., Münch, R., Hohn, M. J., Jahn, D., and Söll, D. (2005a). Nanoarchaeum equitans creates functional tRNAs from separate genes for their $5^{\prime}$ - and $3^{\prime}$ halves. Nature 433, 537-541. doi: 10.1038/nature03233

Randau, L., Pearson, M., and Söll, D. (2005b). The complete set of tRNA species in Nanoarchaeum equitans. FEBS Lett. 579, 2945-2947. doi: 10.1016/j.febslet.2005.04.051

Randau, L., and Söll, D. (2008). Transfer RNA genes in pieces. EMBO Rep. 9, 623-628. doi: 10.1038/embor.2008.101

Reinhold-Hurek, B., and Shub, D. A. (1992). Self-splicing introns in tRNA genes of widely divergent bacteria. Nature 357, 173-176. doi: 10.1038/357173a0

Reyes, V. M., and Abelson, J. (1988). Substrate recognition and splice site determination in yeast tRNA splicing. Cell 55, 719-730. doi: 10.1016/00928674(88)90230-9

Rogers, H. H., and Griffiths-Jones, S. (2014). tRNA anticodon shifts in eukaryotic genomes. RNA 20, 269-281. doi: 10.1261/rna.041681.113

Salinas, T., Duby, F., Larosa, V., Coosemans, N., Bonnefoy, N., Motte, P., et al. (2012). Co-evolution of mitochondrial tRNA import and codon usage determines translational efficiency in the green alga chlamydomonas. PLoS Genet. 8:e1002946. doi: 10.1371/journal.pgen.1002946

Schimmel, P., Giegé, R., Moras, D., and Yokoyama, S. (1993). An operational RNA code for amino acids and possible relationship to genetic code. Proc. Natl. Acad. Sci. U.S.A. 90, 8763-8768. doi: 10.1073/pnas.90.19.8763

Schimmel, P., and Ribas de Pouplana, L. (1995). Transfer RNA: from minihelix to genetic code. Cell 81, 983-986. doi: 10.1016/S0092-8674(05)80002-9

Soma, A., Onodera, A., Sugahara, J., Kanai, A., Yachie, N., Tomita, M., et al. (2007). Permuted tRNA genes expressed via a circular RNA intermediate in Cyanidioschyzon merolae. Science 318, 450-453. doi: 10.1126/science. 1145718

Soma, A., Sugahara, J., Onodera, A., Yachie, N., Kanai, A., Watanabe, S., et al. (2013). Identification of highly-disrupted tRNA genes in nuclear genome of the red alga, Cyanidioschyzon merolae 10D. Sci. Rep. 3:2321. doi: 10.1038/ srep02321

Song, J., and Markley, J. L. (2007). Three-dimensional structure determined for a subunit of human tRNA splicing endonuclease (Sen15) reveals a novel dimeric fold. J. Mol. Biol. 366, 155-164. doi: 10.1016/j.jmb.2006.11.024

Sugahara, J., Fujishima, K., Morita, K., Tomita, M., and Kanai, A. (2009). Disrupted tRNA gene diversity and possible evolutionary scenarios. J. Mol. Evol. 69, 497-504. doi: 10.1007/s00239-009-9294-6

Sugahara, J., Fujishima, K., Nunoura, T., Takaki, Y., Takami, H., Takai, K., et al. (2012). Genomic heterogeneity in a natural archaeal population suggests a model of tRNA gene disruption. PLoS ONE 7:e32504. doi: 10.1371/journal.pone.0032504

Sugahara, J., Kikuta, K., Fujishima, K., Yachie, N., Tomita, M., and Kanai, A. (2008). Comprehensive analysis of archaeal tRNA genes reveals rapid increase of tRNA introns in the order thermoproteales. Mol. Biol. Evol. 25, 2709-2716. doi: $10.1093 / \mathrm{molbev} / \mathrm{msn} 216$ 
Sugahara, J., Yachie, N., Arakawa, K., and Tomita, M. (2007). In silico screening of archaeal tRNA-encoding genes having multiple introns with bulge-helix-bulge splicing motifs. RNA 13, 671-681. doi: 10.1261/rna.309507

Sugahara, J., Yachie, N., Sekine, Y., Soma, A., Matsui, M., Tomita, M., et al. (2006). SPLITS: a new program for predicting split and intron-containing tRNA genes at the genome level. In Silico Biol. (Gedrukt) 6, 411-418.

Sun, F.-J., and Caetano-Anollés, G. (2007). The origin and evolution of tRNA inferred from phylogenetic analysis of structure. J. Mol. Evol. 66, 21-35. doi: 10.1007/s00239-007-9050-8

Tamura, K., and Schimmel, P. (2004). Chiral-selective aminoacylation of an RNA minihelix. Science 305, 1253-1253. doi: 10.1126/science.1099141

Tanaka, T., and Kikuchi, Y. (2001). Origin of the cloverleaf shape of transfer RNAthe double-hairpin model: implication for the role of tRNA intron and the long extra loop. Viva Origino. 29, 134-142.

Tang, T. H., Rozhdestvensky, T. S., d'Orval, B. C., Bortolin, M.-L., Huber, H., Charpentier, B., et al. (2002). RNomics in Archaea reveals a further link between splicing of archaeal introns and rRNA processing. Nucleic Acids Res. 30, 921-930. doi: 10.1093/nar/30.4.921

Tanner, M., and Cech, T. (1996). Activity and thermostability of the small selfsplicing group I intron in the pre-tRNA(lle) of the purple bacterium Azoarcus. RNA 2, 74-83.

Thompson, L. D., and Daniels, C. J. (1990). Recognition of exon-intron boundaries by the Halobacterium volcanii tRNA intron endonuclease. J. Biol. Chem. 265, 18104-18111.

Tocchini-Valentini, G., Saks, M. E., and Abelson, J. (2000). tRNA leucine identity and recognition sets. J. Mol. Biol. 298, 779-793. doi: 10.1006/jmbi.2000.3694

Tocchini-Valentini, G. D., Fruscoloni, P., and Tocchini-Valentini, G. P. (2005a). Coevolution of tRNA intron motifs and tRNA endonuclease architecture in Archaea. Proc. Natl. Acad. Sci. U.S.A. 102, 15418-15422. doi: 10.1073/pnas.0506750102

Tocchini-Valentini, G. D., Fruscoloni, P., and Tocchini-Valentini, G. P. (2005b). Structure, function, and evolution of the tRNA endonucleases of Archaea: an example of subfunctionalization. Proc. Natl. Acad. Sci. U.S.A. 102, 8933-8938. doi: $10.1073 /$ pnas. 0502350102

Tocchini-Valentini, G. D., and Tocchini-Valentini, G. P. (2012). Avatar pre-tRNAs help elucidate the properties of tRNA-splicing endonucleases that produce tRNA from permuted genes. Proc. Natl. Acad. Sci. U.S.A. 109, 21325-21329. doi: 10.1073/pnas. 1219336110

Trotta, C. R., Miao, F., Arn, E. A., Stevens, S. W., Ho, C. K., Rauhut, R., et al. (1997). The yeast tRNA splicing endonuclease: a tetrameric enzyme with two active site subunits homologous to the archaeal tRNA endonucleases. Cell 89, 849-858. doi: 10.1016/S0092-8674(00)80270-6

Turk, R. M., Chumachenko, N. V., and Yarus, M. (2010). Multiple translational products from a five-nucleotide ribozyme. Proc. Natl. Acad. Sci. U.S.A. 107, 4585-4589. doi: 10.1073/pnas.0912895107
Vogel, J., and Hess, W. R. (2001). Complete $5^{\prime}$ and $3^{\prime}$ end maturation of group II intron-containing tRNA precursors. RNA 7, 285-292. doi: 10.1017/S1355838201001960

Weiner, A. M., and Maizels, N. (1999). The genomic tag hypothesis: modern viruses as molecular fossils of ancient strategies for genomic replication, and clues regarding the origin of protein synthesis. Biol. Bull. 196, 327. doi: $10.2307 / 1542962$

Widmann, J., Giulio, M. D., Yarus, M., and Knight, R. (2005). tRNA creation by hairpin duplication. J. Mol. Evol. 61, 524-530. doi: 10.1007/s00239-004-0315-1

Williams, T. A., Foster, P. G., Cox, C. J., and Embley, T. M. (2013). An archaeal origin of eukaryotes supports only two primary domains of life. Nature 504, 231-236. doi: 10.1038/nature12779

Wolf, Y. I., Aravind, L., Grishin, N. V., and Koonin, E. V. (1999). Evolution of aminoacyl-tRNA synthetases-analysis of unique domain architectures and phylogenetic trees reveals a complex history of horizontal gene transfer events. Genome Res. 9, 689-710. doi: 10.1101/gr.9.8.689

Wuchty, S., Fontana, W., Hofacker, I. L., and Schuster, P. (1999). Complete suboptimal folding of RNA and the stability of secondary structures. Biopolymers 49, 145-165. doi: 10.1002/(SICI) 1097-0282(199902)49:2<145::AID-BIP4> 3.0.CO;2-G

Yokobori, S.-I., Itoh, T., Yoshinari, S., Nomura, N., Sako, Y., Yamagishi, A., et al. (2009). Gain and loss of an intron in a protein-coding gene in Archaea: the case of an archaeal RNA pseudouridine synthase gene. BMC Evol. Biol. 9:198. doi: 10.1186/1471-2148-9-198

Yoshinari, S., Shiba, T., Inaoka, D.-K., Itoh, T., Kurisu, G., Harada, S., et al. (2009). Functional importance of Crenarchaea-specific extra-loop revealed by an X-ray structure of a heterotetrameric crenarchaeal splicing endonuclease. Nucleic Acids Res. 37, 4787-4798. doi: 10.1093/nar/gkp506

Conflict of Interest Statement: The authors declare that the research was conducted in the absence of any commercial or financial relationships that could be construed as a potential conflict of interest.

Received: 03 April 2014; paper pending published: 14 April 2014; accepted: 28 April 2014; published online: 26 May 2014.

Citation: Fujishima K and Kanai A (2014) tRNA gene diversity in the three domains of life. Front. Genet. 5:142. doi: 10.3389/fgene.2014.00142

This article was submitted to Non-Coding RNA, a section of the journal Frontiers in Genetics.

Copyright (c) 2014 Fujishima and Kanai. This is an open-access article distributed under the terms of the Creative Commons Attribution License (CC BY). The use, distribution or reproduction in other forums is permitted, provided the original author(s) or licensor are credited and that the original publication in this journal is cited, in accordance with accepted academic practice. No use, distribution or reproduction is permitted which does not comply with these terms. 